\title{
LIPOHIPERTROFIA: CONOCIMIENTO Y EDUCACIÓN AL TRATAMIENTO CON INSULINA EN LA DIABETES MELLITUS
}

\author{
SANDRA MILENA CANO IBARRA¹, GLORIA LASTRE AMELL , MARÍA ALEJANDRA ORÓSTEGUI SANTANDER²
}

${ }^{1}$ Enfermera. Doctora en Ciencia, mención: gerencia. Magister en Enfermería. Especialista en Salud Ocupacional. Docente e investigadora. Universidad Simón Bolívar, Barranquilla, Colombia. orcid.org/0000-0003-0153-1615

${ }^{2}$ Enfermera. Magister en Salud Pública. Docente e investigadora. Universidad Simón Bolivar, Barranquilla, Colombia. orcid.org/0000-0002-8855-3931

${ }^{3}$ Enfermera. Doctorante en Educación. Magister en Educación. Directora programa de Enfermería e investigadora. Universidad Simón Bolívar, Barranquilla, Colombia. orcid.org/0000-0003-2218-6734

Correspondencia: Sandra Milena Cano Ibarra, calle 79 No. 42-329, edificio Villa del jardín apartamento 4G, teléfono 301-5019859, Barranquilla Atlántico, Colombia. scano1@unisimonbolivar.edu.co

Recibido: 23 de noviembre de 2016 Aceptado: 25 de abril de 2017

\section{Resumen}

Introducción: la diabetes mellitus tipo 1 es una enfermedad crónica que, una vez diagnósticada, genera cambios en el estilo de vida del paciente. Para lograr un tratamiento óptimo y evitar complicaciones posteriores, como lipohipertrofia por el aumento en el uso de la insulina, es de vital importancia que se brinde a los pacientes y el personal médico una adecuada educación, para que adquieran conocimientos y habilidades en la aplicación de la inyección de insulina. La lipohipertrofia se presenta por acumulación de grasa subcutánea en el sitio donde se inyecta constantemente la insulina. Objetivo: describir la lipohipertrofia en pacientes con diabetes mellitus, los conocimientos y educación que debe adquirir con respecto al tema. Metodología: se realizó una revisión de literatura en las bases de datos SciELO, Ovid, Medline, PubMed, ScienceDirect y Elsevier, que permitió identificar 71 artículos, de los cuales 50 cumplían con los criterios y pertinencia de la búsqueda. Resultados: se seleccionó la información más completa y pertinente de las bases de datos, respecto a la lipohipertrofia, conocimiento, educación y tratamiento con insulina en la diabetes Mellitus. Conclusión: esta revisión permitió determinar que la lipohipertrofia, una complicación secundaria del tratamiento con insulina en la diabetes Mellitus; se origina en factores tales como la técnica de aplicación y rotación inadecuadas de los puntos de inyección, el escaso conocimiento de los pacientes acerca del tratamiento y la falta de educación del personal sanitario con respecto al uso de las técnicas de aplicación de la insulina.

Palabras clave: hipertrofia; conocimiento; educación; diabetes mellitus; insulina.

\section{LIPOHYPERTROPHY: KNOWLEDGE AND EDUCATION TO TREATMENT WITH INSULIN IN DIABETES MELLITUS}

\section{Summary}

Introduction: Diabetes mellitus type 1 is a chronic disease that, once diagnosed, generates changes in the patient's lifestyle. In order to achieve optimal treatment and avoid subsequent complications, such as lipohypertrophy caused by the increase in the use of insulin, it is vital that patients and medical personnel receive adequate education, so that they acquire knowledge and skills related to the application of the insulin injection. Lipohypertrophy occurs due to the accumulation of subcutaneous fat at the site where insulin is repeatedly injected. Objective: To describe lipohy- 
pertrophy in patients with diabetes mellitus, as well as the knowledge and education that should be acquired on the subject. Methodology: A review of the literature in the databases SciELO, Ovid, Medline, PubMed, ScienceDirect, and Elsevier was carried out, from which 71 articles we identified, and of which 50 met the criteria and were relevant to the search. Results: The most complete and pertinent information was selected from the databases regarding lipohypertrophy, knowledge, education, and insulin treatment in diabetes mellitus. Conclusion: This research allowed us to determine that lipohypertrophy, a secondary complication of insulin treatment in diabetes mellitus, is caused by factors such as an inadequate application technique and inadequate rotation of the injection sites, as well as the lack of knowledge that patients have regarding the treatment, and the lack of education of health personnel when it comes to the use of insulin application techniques.

Keywords: hypertrophy; knowledge; education; Mellitus diabetes; insulin..

\title{
LIPOHIPERTROFIA: CONHECIMENTO E EDUCAÇÃO AO TRATAMENTO COM INSULINA NA DIABETE MELLITUS
}

\begin{abstract}
Resumo
Introdução. A diabetes mellitus tipo 1 é uma doença crônica que, uma vez diagnosticada, gera mudanças no estilo de vida do paciente. Para obter um tratamento ótimo e evitar complicações posteriores, como lipohipertrofia pelo aumento no uso da insulina, é de vital importância proporcionar aos pacientes e o pessoal médico uma adequada educação, para que adquiram conhecimentos e habilidades na aplicação da injeção de insulina. A lipohipertrofia se apresenta por acumulação de gordura subcutânea no lugar onde se injeta constantemente a insulina. Objetivo: descrever a lipohipertrofia em pacientes com diabetes mellitus, os conhecimentos e educação que deve adquirir com respeito ao tema. Metodologia: se realizou una revisão de literatura nas bases de dados SciELO, Ovid, Medline, PubMed, ScienceDirect, Elsevier, que permitiu identificar 71 artigos, dos quais 50 cumpriam com os critérios e pertinência da busca. Resultados: selecionou-se a informação mais completa e pertinente das bases de dados, com relação à lipohipertrofia, conhecimento, educação e tratamento com insulina na diabetes Mellitus. Conclusão: esta revisão permitiu determinar que a lipohipertrofia, uma complicação secundária do tratamento com insulina na diabetes Mellitus, origina-se em fatores tais como a técnica de aplicação e rotação inadequadas dos pontos de injeção, o escasso conhecimento dos pacientes sobre o tratamento e a falta de educação do pessoal sanitário com relação ao uso das técnicas de aplicação da insulina.
\end{abstract}

Palavras-chave: hipertrofia; conhecimento; educação; diabetes mellitus; insulina.

\section{Introducción}

La diabetes mellitus (DM) se convierte en un gran desafío para las personas que la padecen, pero no tanto por la enfermedad misma sino por el desconocimiento de las consecuencias y complicaciones puede desarrollar en el transcurso de su vida y que no están directamente derivadas de la enfermedad. Por ejemplo, la diabetes mellitus tipo 1 (DM1), padecimiento más frecuente a edad temprana, preferentemente en niños y adolescentes, se caracteriza por requerir insulina diariamente. El UK Prospective Diabetes Study (UKPDS) demostró que más del $50 \%$ de las personas que padecen de diabetes mellitus tipo 2 necesita casi siempre adicionar insulina a su tratamiento, por lo que es necesario que los pacientes con esta patología empleen una adecuada técnica de inyección, para prevenir la formación de lipohipertrofia (LH) (1). Además, cada vez, más personas con diabetes mellitus tipo 2 (DM2) ven que la insulina se suma a su medicación hipoglucemiante o que la sustituye completamente. La introducción de la insulina humana en los tratamientos ha reducido la aparición de lesiones en la piel lipohipertrófica. Pero la técnica de inyecciones repetitivas en la misma zona, la reutilización de agujas, la profundidad de la aplicación de insulina y la insuficiente desinfección de la piel generan riesgo de desarrollar este tipo de lesiones 
(2). Así pues, el propósito de este artículo es revisar la literatura en relación con la lipohipertrofia desarrollada por pacientes con DM y determinar cuáles son los conocimientos y educación sobre su tratamiento con insulina que deben poseer los pacientes y el personal de salud.

\section{Metodología}

Se realizó una revisión de la literatura a través de las bases de datos bibliográficas PubMed, Medline, ScienceDirect, ScienceOpen, Elsevier, PubReader, MDedge y otras pulicaciones. La revisión se hizo con los descriptores de salud para la búsqueda de lipohipertrofia, diabetes mellitus, insulina, conocimiento de la diabetes y educación al paciente. Se obtuvieron 71 fuentes bibliográficas pertinentes. Acto seguido, las autoras dieron lectura por separado a los resúmenes de los artículos y luego, en conjunto, hicieron una selección de aquellos que se consideraron más relevantes y que abordaban el tema de acuerdo con los propósitos de la investigación. Finalmente, se determinó que 50 estudios cumplían todos los requisitos necesarios para el análisis. Se trata de materiales publicados desde 1984 hasta 2015 (la tabla 1 muestra la estrategia de búsqueda y proceso de selección de los estudios sobre DM y educación al paciente y personal médico).

Tabla 1. Estrategia de búsqueda y proceso de selección

\begin{tabular}{|l|c|}
\hline Artículos & No. \\
\hline Revisados & 71 \\
\hline Seleccionados & 49 \\
\hline Excluidos & 22 \\
\hline Libros & 3 \\
\hline Bases de datos & \\
\hline PubMed & 32 \\
\hline PubReader & 1 \\
\hline Elsevier & 1 \\
\hline Medline & 1 \\
\hline MDedge & 1 \\
\hline Theime & 1 \\
\hline ScienceOpen & 1 \\
\hline ScienceDirect & 2 \\
\hline IBIMA publishing & 2 \\
\hline Revistas no indexadas & 7 \\
\hline Libros (luego del análisis) & 1 \\
\hline
\end{tabular}

\section{Resultados}

A continuación, se describen la lipohipertrofia, los conocimientos y educación que deben tener los pacientes con DM con respecto al tema de investigación, determinados luego de la revisión de literatura.

\section{Lipohipertrofia, causas y desarrollo}

La LH se desarrolla a cualquier edad y en cualquier sitio de aplicación de la inyección, como producto de las inyecciones frecuentes, la duración del tratamiento, una inapropiada técnica de inyección, calidad deficiente de la aguja y las propiedades fisicoquímicas de las insulinas y la respuesta individual. El tamaño de la $\mathrm{LH}$ varía en un amplio intervalo que al inicio puede ser muy doloroso para el paciente (3-5).

En la actualidad, la lipohipertrofia es reconocida como una complicación de la terapia con insulina. La aplicación de insulina en un sitio con LH puede dar lugar a la absorción errática del fármaco, lo que posibilita un mal control de la glucemia. El uso adecuado de la técnica de rotación de la inyección puede reducir la formación de $\mathrm{LH}$, pero no la previene totalmente (6).

Estudios realizados sobre esta temática coinciden en que, en algunos pacientes, la insulina puede generar efectos no deseados como reacciones alérgicas. Vale mencionar que estos efectos han disminuido a menos del 1,0\% desde que se introdujo la insulina humana recombinante; la lipoatrofia y lipohipertrofia aún siguen siendo las complicaciones más frecuentes, debidas a la administración de insulina $(7,8)$. La segunda adquiere importancia particular, debido a que se han notificado casos de absorción irregular y, por tanto, los niveles de glucemia pueden volverse fluctuantes (8-11).

Para tomar correctivos es necesario tener como referencia estudios de investigación con monitorización continua de glucosa y que hayan obtenido resultados significativos entre las diferencias diarias de glucosa y la gravedad de sitio de la inyección con LH (12). Aunque se ha investigado poco sobre la absorción de insulina a partir del tejido lipohipertrófico, se ha determinado que la absorción de insulina isófana (NPH) es marcadamente defectuoso en los sitios de inyección anormales (13) y que la absorción de la insulina regular (Actrapid) se retrasa, determinada por el aclaramiento 125I-insulina (14). Este análisis demuestra la magnitud del problema que existe en el tratamiento y también que es de importancia clínica, debido 
a que las formulaciones con la insulina dan paso a los análogos de insulina (15-18).

Perciun (19), en su reciente investigación para diagnósticar distrofias locales relacionadas con insulina y su dinámica, por medio de un ultrasonido (US) subcutáneo (SC) y comparando de datos clínicos, utilizó la técnica de los US, que se usa para mejorar la técnica de inyección de insulina, mediante la identificación del espesor real de cutis/hipodermis (CST, por sus siglas en inglés). El examen clínico puso en evidencia tres tipos de distrofia locales: hipertrofia, bultos nodulares y atrofia. Esta técnica permitió confirmar los hallazgos clínicos y, por otra parte, diagnósticar la localización, tamaño, profundidad y estructura eco. Al ser un término comparativo, así como una condición para la mejora de la técnica de inyección, el mapeo de CST normal podría ser también parte de una educación específica continua de los pacientes tratados con insulina. Vale tener en cuenta también que un estudio realizado anteriormente por el autor había permitido evaluar los resultados de inyecciones de insulina por medio de un aspecto ecográfico del tejido subcutáneo (20).

\section{Prevalencia y factores asociados con lipohipertrofia}

La prevalencia de la diabetes en la población mundial está en aumento. La disparidad en la disponibilidad y asequibilidad de la atención de la diabetes, así como el bajo conocimiento de la enfermedad y la falta de un buen control de la glucemia aumentan la carga de la enfermedad y, por ende, a la aparición temprana de complicaciones relacionadas con el uso de la insulina (21). Este tipo de complicación dermatológica, como la LH, en los sitios de administración de insulina, es común en los niños con diabetes tipo 1. Estas complicaciones pueden ser dolorosas y deformantes y pueden influir en el control glucémico de los pacientes (22).

De acuerdo Van Munster et al., existen estudios que se hicieron para detectar la presencia de $\mathrm{LH}$, por inspección y palpación, en los que se tuvieron en cuenta las últimas 4 lecturas de los niveles de hemoglobina glucosilada $(\mathrm{HbA} 1 \mathrm{c})$ y otros factores que pueden afectar la $\mathrm{LH}$. En ellos se demostró que todos los pacientes con grados 1 y 2 de LH tenían niveles de HbA1c similares a los de pacientes sin lipohipertrofia, pero esta se relaciona de manera significativa con la dosis de unidades de insulina por kilogramo de peso corporal. La LH se relaciona también con la duración de la diabetes; el lavado de las manos antes de las inyecciones; tipo de insulina; con el número de inyecciones por día; la frecuencia de cambio de la aguja; la persona que pone la inyección; el historial de atopia y, finalmente con el grado de educación de la madre del menor don diabetes (23). A esto se asocia el índice de masa corporal, por lo que los niños con LH necesitan una dosis significativamente mayor de unidades de insulina por kilogramo de peso corporal para lograr un control adecuado en comparación con los niños sin LH (24).

Asimismo, también se ha estimado la prevalencia de la LH en pacientes con DMT2 tratados con insulina, donde se identificaron factores de riesgo para el desarrollo de la LH, como la duración de la diabetes, la longitud de la aguja, la duración de la terapia con insulina, la falta de rotación sistemática de los sitios de inyección y un control insuficiente de la glucemia (25).

Por lo anterior, durante décadas, la lipohipertrofia ha sido reconocida como una complicación propia de la terapia con insulina y su prevalencia sigue siendo alta, a pesar de los avances en la tecnología médica: alrededor del $20-30 \%$ de los pacientes con DMT1, y alrededor de un $4 \%$ de los pacientes con DMT2, desarrollan lipohipertrofia (26). Factores como la edad, el sexo, el nivel de educación, el índice de masa corporal (IMC), el tipo de diabetes, el período de uso de la insulina y el lugar de la inyección influyen significativamente en el desarrollo de lipodistrofia inducida por insulina (27).

Autores como Ji J. y Lou Q (28) refieren que la eficacia de la terapia de inyección en la diabetes depende de la técnica correcta de inyección, la cual los profesionales de salud debemos conocer, para poder proporcionar a los pacientes una adecuada orientación. A fin, por ejemplo, de evitar que los pacientes que sigan inyectándose en zonas con LH, lo que frecuentemente les produce sangrado y moretones, que, además, demuestran que existe relación significativa entre la frecuencia de reutilización de agujas y la lipohipertrofia.

Cabe destacar que el control glucémico deficiente, con niveles erráticos de glucosa en sangre, que se manifiesta como la hiperglucemia recurrente, con episodios impredecibles de hipoglucemia es un problema que aún se enfrenta hoy en la práctica clínica diaria. Las causas incluyen el mal desempeño o el fracaso de hipoglucemiante oral. A pesar de estar en tratamiento con insulina $(38 \%$ de los pacientes con diabetes tipo 2 que requieren tratamiento con insulina después de 10 años) por DMT1 
los pacientes diabéticos siguen teniendo concentración fluctuosa de glucosa. El control inadecuado de la glucemia en los pacientes tratados con insulina se debe a lipohipertrofia inducida por la insulina (26). A lo anterior, se suma el trauma mecánico de las inyecciones, el criotrauma de la insulina refrigerada y la mediación inmune (29).

Un estudio comparativo llevado a cabo con pacientes con DTM1, en el que se utilizaron simultáneamente sensores Dexcom G4 Platinum en uno con lipohipertrofia y otro con tejido normal, durante 2 semanas, dio como resultado una diferencia relativa en los sensores del tejido con LH, mostrando exactitud igual o ligeramente superior que los sensores en tejido normal, en los que fue evidente en todos rangos de glucosa, con una media general relativa de $10,0 \%$ para los sensores en el tejido lipohipertrófico (30).

En cuanto al uso de sensores, existen dudas sobre si estos causan daño a la piel o al tejido subcutáneo, debido a las inserciones repetidas en la misma zona, puesto que la punta del sensor podría inducir traumatismo local agudo y posiblemente más reacciones crónicas de la piel (31). Por lo tanto, se sugiere seguir trabajando para cuantificar los riesgos potenciales del uso de sensores en las zonas de LH durante períodos más largos (32).

La prevalencia de la $\mathrm{LH}$ ha aumentado tanto que no puede ignorarse. Por lo que el profesional de enfermería debe esforzarse para examinar a cada persona que utiliza insulina en su tratamiento. Cuando una persona inicia tratamiento, parte importante de la educación que debe recibir es destacar la importancia de utilizar los materiales y técnica correcta de la inyección (33).

\section{Conocimiento a la asistencia del paciente diabético}

De acuerdo con los informes para el mejoramiento de la salud del paciente diabético, se pueden encontrar avances en cuanto a tecnologías nuevas y necesarias, en los cuales el paciente debe tener todo el conocimiento acerca de las nuevas disposiciones de la asistencia al paciente con tratamiento insulínico.

En la actualidad, existe una cantidad creciente de tecnología al alcance de estos pacientes, tales como los cambios introducidos en los medidores de glucemia capilar, resumidos fundamentalmente en mayor rapidez y necesidad de muestra menor (34).
En consecuencia, la terapia con infusores de insulina se convierte en una alternativa terapéutica para tener en cuenta. El tratamiento con infusión subcutánea continua de insulina es actualmente la mejor vía fisiológica para reemplazar tanto el componente basal de la secreción de insulina, como los picos de secreción insulínica en respuesta a la ingesta. En el momento actual, este tipo de terapia es calificada como segura y resulta ser una buena alternativa de tratamiento en adultos, adolescentes y niños con DM (35).

Otro aspecto de creciente interés por este campo es la introducción de las modernas tecnologías de la comunicación que proveen una mejor calidad asistencial, entre estas podemos mencionar la telemedicina que combina las telecomunicaciones y tecnologías de la información para proporcionar servicios sanitarios independientemente de la localización física de proveedores, pacientes, historias clínicas o equipos. Los nuevos conceptos de gestión sanitaria buscan ofrecer servicios novedosos como la teleconsulta o el telecuidado, que proporcionan ventajas en el acceso a los servicios sanitarios, en la calidad del cuidado proporcionado, en el coste de la provisión de servicios sanitarios o en la educación de las personas implicadas en la asistencia del paciente. Por lo tanto, el objetivo final es la consecución del cuidado compartido y, a la vez, potenciar el papel del paciente en el autocuidado, por medio de una autonomía supervisada (36).

En el avance de nuevos estudios y tecnología realizados en los últimos años, se han identificado importantes temas relacionados con la inyección, la longitud y calibre de la aguja, el IMC, la piel y el espesor del tejido subcutáneo, la resuspensión de insulinas nubladas, las fugas, la elección y rotación del lugar de la inyección, el pellizcar un pliegue de piel y la lipohipertrofia. Por lo que es de gran interés revisar las investigaciones actuales y hacer comparaciones en relación con la terapia de inyección de insulina, para así proporcionar información de traslación práctica sobre la técnica de inyección, las técnicas de enseñanza y aprendizaje específicas para la administración de insulina, y las implicaciones para la educación y el apoyo de autocontrol de la DM (37).

Los últimos avances aportan conocimiento de cómo se debe llevar un apropiado tratamiento. Se recomienda, por ejemplo, el uso de agujas cortas para reducir al mínimo las inyecciones intramusculares involuntarias que pueden aumentar la formación de $\mathrm{LH}$. Al respecto, ac- 
tualmente, la aguja más segura para los niños parece ser la de la pluma de $4 \mathrm{~mm}$; sin embargo, cuando se utiliza en niños de 2-6 años, se debe utilizar la técnica de pliegue, conocida también como piel pellizcada (38).

En otra revisión en relación con el tema, se encontró que la rotación correcta del sitio de la inyección es el factor fundamental en la prevención de LH. La rotación correcta se asocia con una reducción de la variabilidad de la glucosa, la hipoglucemia, el consumo de insulina y los costos del tratamiento (39).

Por otra parte, aportes de otros autores recomiendan utilizar la aguja PEN de $4 \mathrm{~mm}$ para evitar la inyección intramuscular, asegurar un buen control glucémico y, por ende, evitar la LH. En ellos, se concluye que es importante utilizar este tipo de aguja para la administración del tratamiento con insulina en los pacientes que la requie$\operatorname{ran}(40,41)$.

\section{Educación para el paciente con tratamiento insulínico}

Estudios recientes hacen diversas recomendaciones en cuanto a la técnica de inyección de los pacientes con diabetes, asimismo ofrecen recomendaciones específicas para profesionales de la salud. En ese sentido, este artículo proporciona información de revisión de estudios basados en la evidencia en relación con la técnica de inyección y la teoría de la enseñanza y aprendizaje de la mejor técnica. Los educadores en diabetes necesitan reevaluar el modo en que proporcionan instrucciones sobre la administración de insulina y otros medicamentos inyectables.

Investigaciones en relación con la piel y el espesor subcutáneo revelan que las agujas cortas pueden ser apropiadas para la mayoría de los pacientes, independientemente de su IMC. Además, la reevaluación periódica de la técnica de inyección, incluyendo la suspensión de insulinas nubladas y la inspección de los lugares de inyección para lipohipertrofia, es un aspecto crítico del papel del educador en diabetes (42).

La LH, hoy por hoy, es un problema común en los pacientes tratados con insulina. Por lo que se necesitan más esfuerzos para educar a los pacientes y al personal de salud en cuanto a intervenciones simples, como el uso de agujas cortas y la rotación frecuente de los sitios de inyección de insulina, a fin de evitar la LH y mejorar el control glucémico (43).

A pesar de los avances en la tecnología, la LH inducida por insulina sigue siendo una causa común, pero ignorada, del control glucémico insuficiente desde tiempos pasados, por lo cual no debemos permitir que esta situación siga presentándose. Todos los pacientes deben ser examinados cuidadosamente para detectar $\mathrm{LH}$ durante la revisión clínica diabética, mediante inspección y palpación de los puntos de inyección. La educación para los médicos, los enfermeros y los pacientes sobre el reconocimiento y el tratamiento de la $\mathrm{LH}$ es aconsejable (26), por lo que se debe prestar atención cuidadosa a la enseñanza de métodos correctos de la técnica de inyección de insulina (44).

\section{Recomendaciones para evitar la aparición de lipohipertrofia}

Dentro de los pilares fundamentales en el tratamiento de la diabetes las inyecciones requieren de una técnica adecuada que garantice el ingreso de la insulina a los tejidos subcutáneos y, con ello, evitar complicaciones comunes como la LH. Diversas publicaciones señalan pautas acerca de las mejores prácticas de inyección basada en la evidencia (45). Una recomendación importante para mejorar la técnica es utilizar el pliegue cutáneo $(46,47)$.

Ahora bien, a pesar de estar utilizando los nuevos análogos de insulina, se presentan muchos casos de LH. Para resolver este problema, en primera instancia, puede ser suficiente cambiar el sitio de la inyección, pero esto no garantiza que no se desarrolle LH posteriormente. Por lo anterior, se recomienda la inspección de los lugares de inyección en cada visita al médico, lo que ayudará a la detección temprana de esta complicación $(29,48)$.

Por otro lado, es de gran importancia que los educadores reevalúen la forma en que proporcionan instrucciones para la administración de la insulina. A su vez, es necesario crear conciencia de que es un proceso continuo, en el que se debe reevaluar periódicamente la técnica de inyección e inspeccionar las zonas de aplicación de la insulina (42). Hasta donde sabemos, además de la reacción inmune local a los cristales de insulina, el uso frecuente de la misma aguja, la falta de rotación de los puntos de inyección y el uso de los pliegues de la piel, favorecen el desarrollo de LH en los pacientes $(48,49)$. Por lo ante- 
rior, y reconociendo que la administración de la insulina es a menudo difícil, la prevención es vital y puede lograrse mediante la educación en la enfermedad y sobre las mejores técnicas de inyección desde el momento mismo de su diagnóstico (50).

\section{Conclusiones}

La literatura médica nos muestra que la prevalencia de la LH es cada día más frecuente en el paciente diabético, por lo cual debe prestarse más atención a esta complicación. Del mismo modo, la revisión del tema permitió identificar que deben buscarse nuevas estrategias educativas para la prevención de la LH; sin embargo, en los estudios publicados entre los años 2012 y 2015, se evidencian coincidencias con estudios anteriores, en cuanto a definición de la $\mathrm{LH}$, factores de riesgo, posibles causas, técnica de administración y tratamiento de la patología.

Sin embargo, es importante destacar que los últimos estudios apuntan a la importancia de la educación terapéutica, debido a que muchos pacientes, familiares, cuidadores y hasta personal sanitario no poseen conocimientos suficientes acerca de los cuidados, técnica adecuada de aplicación de la inyección y la manera de detectar la LH.

La revisión del tema, sumada a la alta prevalencia en los últimos años de la LH en el paciente diabético, permite concluir que la falta de conocimiento y educación del paciente se debe en gran parte al equipo de salud. Puesto que el rol de los profesionales de estos profesionales es fundamental para un adecuado manejo del tratamiento, ya que una correcta técnica de inyección y la valoración y seguimiento de los sitios utilizados previenen o retardan la aparición de LH. Del mismo modo, es importante implementar estrategias conductuales y psicosociales sobre el tema y que se lleven a cabo nuevos estudios de investigación a fin de mejorar el tratamiento de la DM y patologías relacionadas, como la $\mathrm{LH}$.

\section{Declaración de conflictos de interés}

Las autoras declaran no tener conflicto de intereses en la revisión de literatura, artículos e investigaciones consultadas.

\section{Referencias}

1. UK Prospective Diabetes Study Group. Intensive bloodglucose control with sulphonylureas or insulin compared to conventional treatment and risk of complications in patients with type 2 diabetes (UKPDS 33). Lancet 1998; 352: 837-53.

2. Strauss K, De Gols H, Hannet I, Partanen TM, Frid A. A pan-European epidemiologic study of insulin injection technique in patients with diabetes. Pract Diab Int. 2002; 19(3): 71-6.

3. Volkova N, Davidenko I. Lypohypertrophy in patients receiving insulin therapy: state of the art. Diabetes Mellitus. Diagn Manag Treat. 2011; 2: 80-3.

4. Wallymahmed M, Littler P, Clegg C., Haqqani M. \& MacFarlane, I. A. "Nodules of Fibrocollagenous Scar Tissue Induced by Subcutaneous Insulin Injections: A Cause of Poor Diabetic Control," Postgraduate Medicine Journal, 2004; 80 (950): 732-3.

5. Hofman $\mathrm{P}$, et al. "Defining the Ideal Injection Techniques when Using 5-mm Needles in Children and Adults," Diabetes Care, 2010; 33 (9): 1940-4.

6. Dar I, Dar S, Wani S. Insulin lipohypertrophy: A non-fatal dermatological complication of diabetes mellitus reflecting poor glycemic control. Saudi Journal of Medicine and Medical Sciences, 2013. 1(2): 106-8.

7. Röcken M, Strölin A. Hautkrankenheiten und Diabetes Mellitus. In: Häring HU, Gallwitz B, Müller-Wieland D, Klaus-Henning V, Mehnert H. Diabetologie in Klinik und Praxis. Deutchsland: 6 Auflage; 2011. p. 524-9.

8. Heinemann L. Insulin absorption from lipodystrophic areas: a (neglected) source of trouble for insulin therapy? J Diabetes Sci Technol. 2010; 4(3): 750-3.

9. Behm B, et al. Skin signs in diabetes mellitus. J Eur Acad Dermatol Venereol. 2012; 26(10): 1203-11.

10. Murphy-Chutorian B, Han G, Cohen SR. Dermatologic manifestations of diabetes mellitus: a review. Endocrinol Metab Clin North Am. 2013; 42(4): 869-98.

11. Van Hattem S, Bootsma A, Thio H. Skin manifestations of diabetes. Cleve Clin J Med. 2008; 75(11): 772, 774, 776-7 passim.

12. Alemzadeh R, Loppnow C, Parton E, Kirby M. Glucose sensor evaluation of glycemic instability in pediatric type 1 diabetes mellitus. Diabetes Technol Ther 2003; 5: 167-73.

13. Thow J, Johnson A, Marsden S, Taylor R, Home PD. Morphology of palpably abnormal injection sites and effects on absorption of isophane (NPH) insulin. Diabet Med. 1990; 7: 795-9.

14. Young R, Hannan W, Frier B, Steel J, Duncan L. Diabetic lipohypertrophy delays insulin absorption. Diabetes Care. 1984; 7: 479-80.

15. Griffin M, Feder A, Tamborlane W. Lipoatrophy associated with lispro insulin in insulin pump therapy: an old complication, a new cause? Diabetes Care. 2001; 24(1): 174.

16. Del Olmo M, Campos V, Abellán P, Merino-Torres J, Piñón F. A case of lipoatrophy with insulin detemir. Diabetes Res Clin Pract. 2008; 80(1): e20-1.

17. Ampudia J, Hasbum B, Carmena R. A new case of lipoathrophy with lispro insulin in insulin pump therapy: is 
there any insulin preparation free of complications? Diabetes Care. 2003; 26(3): 953-4.

18. Radermecker R, Piérard G, Scheen A. Lipodystrophy reactions to insulin: effects of continuous insulin infusion and new insulin analogs. Am J Clin Dermatol. 2007; 8(1): 21-8.

19. Perciun R, Mihu M. The Subcutis Ultrasound Map of Type 1 Diabetic Children Improves the Diagnosis of Local Dystrophies and Insulin Injection Technique, Pediatrics Research International Journal, Vol. 2014 (2014). DOI: $10.5171 / 2014.402780$

20. Perciun, R. "Aspecto ecográfico del tejido subcutáneo Distrofias como resultado de inyecciones de insulina," Médico ecografía, 2010; 12 (2): 104-9.

21. Manjula G, Saravana A. Problems Associated with insulin administration among patients with type 2 Diabetes Mellitus. Impact Factor (2013): 4.438.

22. Van Munster, H, et al. Dermatological complications of insulin therapy in children with type 1 diabetes. European Diabetes Nursing. 2014; 11(3): 79-84.

23. Omar M, El-Kafoury A, El-Araby R. Lipohypertrophy in children and adolescents with type 1 diabetes and the associated factors. BMC Research Notes 2011, 4: 290.

24. Annersten M, Wilman A. Performing subcutaneous injections: A literature review. Wordviews on Evid Based Nurs. 2005 [citado 22 MAY 2016]; 2(3): 122-30.

25. Al Ajlouni M, Abujbara M, Batieha A, Ajlouni K. Prevalence of Lipohypertrophy and associated risk factors in insulin-treated patients with type 2 diabetes mellitus. nt $\mathrm{J}$ Endocrinol Metab. 2015 Apr; 13(2): e20776.

26. Malwa G, Balami D, Deshmukh S, Groft M, Bodmer C, Patel M. Insulin-induced Lipohypertrophy, past present and future: are we lose the bottle. Endocrine. 2010; 22: 268.

27. Hajheydari Z, Kashi Z, Akha O, Akbarzadeh S. Frequency of lipodystrophy induced by recombinant human insulin. Eur Rev Med Pharmacol Sci. 2011 Oct; 15(10): 1196-201.

28. Ji J, Lou Q. Insulin pen injection technique survey in patients with type 2 diabetes in mainland China in 2010. 2014 Jun; 30(6): 1087-93.

29. Babiker A, Datta V. Lipoatrophy with insulin analogues in type I diabetes. Arch Dis Child published online June 22, 2010. Doi: $10.1136 /$ adc.2010.183731

30. DeSalvo DJ, Shanmugham S, Ly TT, Wilson DM, Buckingham BA. Accuracy evaluation of blood glucose monitoring systems in childrenon overnight closed-loop control. J Diabetes Sci Technol 2014; 8: 969-73.

31. Heinemann L, Hirsch L, Hovorka R. Lipohy pertrophy and the artificial pancreas: is this an issue? J Diabetes Sci Technol 2014; 8: 915-7.

32. DeSalvo DJ, et al. Effect of Lipohypertrophy on accuracy of continuous glucose monitoring in patients with type 1 diabetes. Diabetes Care. August 25, 2015. pii: dc151267

33. Cunningham MT, McKenna MJ. Lipohypertrophy in insulin-treated diabetes: Prevalence and associated risk factors. Journal of Diabetes Nursing. 2013 17: 340-3.
34. Pettis R, et al. "Intradermal Microneedle Delivery of Insulin Lispro Achieves Faster Insulin Absorption and Insulin Action than Subcutaneous Injection," Diabetes Technology \& Therapeutics, 2011; 13(4): 435-42.

35. Goupta J, Felner E. \& Prausnitz R. "Rapid Pharmacokinetics of Intradermal Insulin Administered Using Microneedles in Type 1 Diabetes Subjects," Diabetes Technology \& Therapeutics, 2011; 13(4): 451-6.

36. Merino J. Nuevas tecnologías en el seguimiento y control del paciente diabético Editorial De La Sociedad Española De Diabetes. 2007.

37. Saltiel-Berzin R, Cypress M, Gibney M. Translating the research in insulin injection technique: implications for practice. Diabetes Educ. 2012 Sep-Oct; 38(5): 635-43.

38. Lo Presti D, Ingegnosi C, Strauss K. Skin and subcutaneous thickness at injecting sites in children with diabetes: ultrasound findings and recommendations for giving injection. 2012 Nov; 13(7): 525-33.

39. Blanco M, Hernández MT, Strauss KW, Amaya M. Prevalence and risk factors of lipohypertrophy in insulin-injecting patients with diabetes. 2013; 39(5): 445-53.

40. Grassi G, Scuntero P, Trepiccioni R, Marubbi F, Strauss $\mathrm{K}$. Optimizing insulin injection technique and its effect on blood glucose control. Journal of Clinical \& Translational Endocrinology. 2014; 1(4): 145-50.

41. Bergenstal RM, et al. Safety and efficacy of insulin therapy delivered via a $4 \mathrm{~mm}$ pen needle in obese patients with diabetes. 2015; 90(3): 329-38.

42. Saltiel-Berzin R, Cypress M, Gibney M. Translating the research in insulin injection technique: implications for practice. Diabetes Educ. 2012; 38(5): 635-43.

43. Al Ajlouni M, Abujbara M, Batieha A, Ajlouni K. prevalence of lipohypertrophy and associated risk factors in insulin-treated patients with type 2 diabetes mellitus. J Endocrinol Metab. 2015; 13(2): e20776.

44. Stanley Landau, F.C.P.(S.A.). Insulin-Induced Lipohypertrophy. N Engl J Med 2012; 366: e9.

45. Frid $A$, et al. New injection recommendations for patients with diabetes. September 2010. 36(s2): s3-s18.

46. Gibney M, Arce, C, Byron K. \& Hirsch L. Skin and Subcutaneous Adipose Layer Thickness in Adults with Diabetes at Sites Used for Insulin Injections: Implications for Needle Length Recommendations. Current Medical Research \& Opinion, 2010; 26(6): 1519-30.

47. Selkow N, Pietrosimone B\& Saliba S. A. Subcutaneous Thigh Fat Assessment: A Comparison of Skinfold Calipers and Ultrasound Imaging. Journal of Athletic Training, 2011; 46 (1): 50-4.

48. Mokta J, Mokta K, Panda P. Insulin lipodystrophy and lipohypertrophy. Indian J Endocrinol Metab. 2013; 17: 773-4.

49. Pledger J, Hicks D, Kirkland F, Down S. Importance of injection technique in diabetes. Journal of Diabetes Nursing; 2012, 16(4): 160.

50. Nagesh V, Kalra S. Type 1 diabetes: Syndromes in resource-challenged settings. J Pak Med Assoc. 2015 Jun; 65(6): 681-5. 